\title{
Characteristics of yak platelet derived growth factors-alpha gene and its expression in brain tissues
}

\author{
Z. Huang, Y. Pan, P. Liu, S. Yu, Y. Cui \\ College of Veterinary Medicine, Gansu Agricultural University, Lanzhou, China
}

\begin{abstract}
Background: Platelet derived growth factors (PDGFs) are key components of autocrine and paracrine signalling, both of which play important roles in mammalian developmental processes. PDGF expression levels also relate to oxygen levels. The characteristics of yak PDGFs, which are indigenous to hypoxic environments, have not been clearly described until the current study.

Materials and methods: We amplified the open reading frame encoding yak (Bos grunniens) platelet derived growth factor-alpha (PDGFA) from a yak skin tissue CDNA library by reverse transcriptase polymerase chain reaction $(P C R)$ using specific primers and Sanger dideoxy sequencing. Expression of PDGFA mRNA in different portions of yak brain tissue (cerebrum, cerebellum, hippocampus, and spinal cord) was detected by quantitative real-time PCR (qRT-PCR). PDGFA protein expression levels and its location in different portions of the yak brain were evaluated by western blot and immunohistochemistry.
\end{abstract}

Results: We obtained a yak PDGFA 755 bp cDNA gene fragment containing a 636 bp open reading frame, encoding 211 amino acids (GenBank: KU851801). Phylogenetic analysis shows yak PDGFA to be well conserved, having 98.1\% DNA sequence identity to homologous Bubalus bubalus and Bos taurus PDGFA genes. However, 8 nucleotides in the yak DNA sequence and 4 amino acids in the yak protein sequence differ from the other two species. PDGFA is widely expressed in yak brain tissue, and furthermore, PDGFA expression in the cerebrum and cerebellum are higher than in the hippocampus and spinal cord ( $p>0.05)$. PDGFA was observed by immunohistochemistry in glial cells of the cerebrum, cerebellum, and hippocampus, as well as in pyramidal cells of the cerebrum, and Purkinje cell bodies of the hippocampus, but not in glial cells of the spinal cord.

Conclusions: The PDGFA gene is well conserved in the animal kingdom; however, the yak PDGFA gene has unique characteristics and brain expression patterns specific to this high elevation species. (Folia Morphol 2017; 76, 4: 551-557)

Key words: yak, platelet derived growth factor-alpha, expression, brain

\section{INTRODUCTION}

Platelet derived growth factors (PDGFs) are synthesized and secreted by a variety of cells, including fibro- blasts, muscle cells, and glial cells [13, 17, 23]. PDGFs combine with tyrosine kinase receptors (platelet-derived growth factor receptors, PDGFRs), have been extensively

Address for correspondence: Dr. Y. Cui, College of Veterinary Medicine, Gansu Agricultural University, No. 1 Yingmen village, Anning District, Lanzhou, Gansu Province, Post code: 730070, P.R. China, tel:+86-0931-7632858, fax: +86-0931-7632858, e-mail: cuiyan369@sina.com 
Table 1. The information of primers used in reverse transcriptase polymerase chain reaction (PCR) and quantitative real-time PCR (qRT-PCR)

\begin{tabular}{|c|c|c|c|c|}
\hline Gene & Sequence $5^{\prime}$ to $3^{\prime}$ & Temperature $\left({ }^{\circ} \mathrm{C}\right)$ & PCR product (bp) & Genbank accession no. \\
\hline PDGFA(1) & $\begin{array}{l}\text { GATGAGGACCTGGGCTTGT } \\
\text { AGACCACGCTGGCAGTAAG }\end{array}$ & 59 & 755 & J0326282/NM001075231 \\
\hline PDGFA(2) & 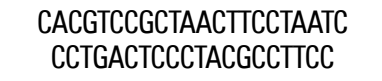 & 60 & 264 & KU851801 \\
\hline GAPDH & $\begin{array}{l}\text { AACCACGAGAAGTATAACAACACC } \\
\text { TCCCTCCACGATGCCAAA }\end{array}$ & 59 & 114 & EU195062 \\
\hline
\end{tabular}

studied in developing organisms $[3,5,10]$, and play key roles in the process of development [1], promotion of cell mitosis, and chemotaxis, but can also induce series of biological effects, such as cell proliferation, differentiation, and the prevention of apoptosis [1, 7].

Yet the physiological roles of PDGFs in adult animals remain poorly understood [1, 4]. All PDGFs function as secreted, disulphide-linked homodimers, but only PDGF-alpha and PDGF-beta (PDGFA and PDGFB) can form functional heterodimers, and have been widely studied. The structure and function of the PDGFA protein are similar to those of vascular endothelial growth factor (VEGF) proteins [2, 8], which are also highly conserved in evolution [19], and contain a cysteine knot motif in common with PDGFs [15]. PDGFRA signalling is not required for the specification of oligodendrocyte progenitors (OPs), but the further proliferation and spreading of OPs in the central nervous system (CNS) depend on PDGFA signalling through PDGFRA [16]. PDGFA drives not only the proliferation of OPs in the embryo, but it also determines the number of OPs in the adult brain [26]. PDGFA is also a critical ligand for PDGFRA in oligodendrocyte development. PDGFA is expressed by neurons and astrocytes throughout the CNS, and is constitutively released from neural cell bodies. Moreover, it is also closely related to pathologies associated with the stress of hypoxic conditions [12, 13].

Domestic yaks (Bos grunniens) on the Qinghai Tibet Plateau are less sensitive to and readily adapt to hypoxia [11, 27]. Undoubtedly the yak brain plays an important role in this high-altitude hypoxia adaptation. Many researchers have previously investigated the characteristics of PDGFA in other animals, and its expression in brain tissue, under hypoxic stress for short periods of time. These studies have often observed the transition to "normal" oxygen levels (i.e. $\mathrm{O}_{2}: 21 \%$ ) after birth. However, yak PDGFAs have not been characterised, despite the yak having lived in hypoxic environments for most of its evolutionary history, yet alone each animal's lifetime. Therefore, our study of the characteristics and expression of yak PDGFA in brain tissue should prove highly informative.

In our study, the characteristics of yak PDGFA were analysed, and its expression in yak brain tissues was studied by quantitative real-time polymerase chain reaction (qRT-PCR), western blot, and immunohistochemistry. Our study should prove helpful for exploring the effects of PDGFA in hypoxia regulatory processes related to physiological function in this Tibet Plateau species.

\section{MATERIALS AND METHODS}

\section{Yak brain sampling}

The brain tissues (cerebrum, cerebellum, hippocampus, and spinal cord) and skin tissue of yak ( $n=5$, 3 years old) were obtained from the Lejiawan slaughterhouse, located in Xining City, Qinghai Province, China. All experimental and surgical procedures were approved by the Biological Studies Animal Care and Use Committee, Qinghai Province, People's Republic of China. Brain tissue samples were fixed using $4 \%$ neutral paraformaldehyde for immunohistochemical staining; other brain samples and skin samples were quick frozen in liquid nitrogen for protein and RNA extraction.

\section{Primer design and PCR amplification}

Bos grunniens glyceraldehyde-3-phosphate dehydrogenase (GAPDH, GenBank: EU195062) was used as a reference control. PDGFA sequences of cattle (Bos taurus, GenBank: NM001075231) and Asian buffalo (Bubalus bubalus, GenBank: JQ326282) served as references for coding region PCR primer design. Those PDGFA coding regions were aligned with the yak genome sequence (NCBI genome assembly: BosGru_v2.0), and conserved upstream and downstream sequences were selected for qRT-PCR primer design with Primer Premier 6.0 (Applied Biosystems) (Table 1). Evolutionary inference and sequence similarity analysis were performed with MEGA 6.0 and DNA Star. 
RNA was extracted from collected skin tissues using an RNA Total Kit (Tiangen Bio, Beijing, China). First strand CDNA synthesis was performed using a reverse transcription kit (TaKaRa Bio, Dalian, China) according to the manufacturer protocol, with oligo-dT primers and approximately $1 \mu \mathrm{g}$ of total RNA as a template. PCR was carried out in a $20 \mu \mathrm{L}$ reaction system containing $1 \mu \mathrm{L}$ cDNA template, $10 \mu \mathrm{L}$ Taq DNA polymerase Master Mix (Promega, USA), $1 \mu \mathrm{L}$ each forward and reverse primer $(0.2 \mu \mathrm{mol} / \mathrm{mL})$, and $\mathrm{ddH} 2 \mathrm{O}$ (up to $20 \mu \mathrm{L}$ ). PCR conditions were as follows: initial denaturation for $5 \mathrm{~min}$ at $95^{\circ} \mathrm{C}, 35$ cycles at $94^{\circ} \mathrm{C}$ for $30 \mathrm{~s}$, annealing temperature of $60^{\circ} \mathrm{C}$ for $10 \mathrm{~s}$, and extension at $72^{\circ} \mathrm{C}$ for $30 \mathrm{~s}$. The final extension step was followed by a $10 \mathrm{~min}$ extension at $72^{\circ} \mathrm{C}$. The PCR products were separated in $1.2 \%$ agarose gel, and then sent for Sanger dideoxy sequencing (The Beijing Genomics Institute, Beijing, China).

\section{Real-time PCR analysis}

Quantitative real-time PCR reactions were performed on an Applied Biosystems ViiA ${ }^{T M} 7$ Real-Time PCR System (Thermo Fisher Scientific Inc., Waltham, MA, USA), with equal amounts of $10 \mu \mathrm{L}$ SYBR Green II Master Mix, $0.4 \mu \mathrm{L}$ Passive Reference Dye II (Takara, Dalian, China), 1.0 $\mu$ Leach forward and reverse primers $(10 \mu \mathrm{mol} / \mathrm{L}), 6.6 \mu \mathrm{L} \mathrm{ddH} 2 \mathrm{O}$, and $1 \mu \mathrm{L}$ cDNA in a final volume of $20 \mu \mathrm{L}$. Standard amplification conditions were $3 \mathrm{~min}$ at $95^{\circ} \mathrm{C}$, and 42 cycles of $10 \mathrm{~s}$ at $95^{\circ} \mathrm{C}$, followed by $32 \mathrm{~s}$ at $60^{\circ} \mathrm{C}$. Afterwards, the corresponding dissociation curves were analysed. Each qRT-PCR run included GAPDH control and target genes, and each sample was repeated 3 times. We analysed the relative expression of mRNA of each gene by quantitative fluorescence, with the level of GAPDH expression being used as an endogenous control, and using the $2^{-\triangle \Delta \mathrm{Ct}}$ method to analyse relative gene expression [21].

\section{Western blot analysis for PDGFA}

Total protein extracted from the brain tissues was obtained using a protein extraction kit (Beyotime Biotechnology, Jiangsu, China) according to the manufacturer's instructions. Protein concentrations were measured by the bovine serum albumin (BSA) method (Pierce, Rockford, IL, USA) using BSA as the standard. The protein samples were diluted to the same concentration. Mixtures of equal amounts $(20 \mu \mathrm{g})$ of protein from each sample in loading buffer were denatured at $100^{\circ} \mathrm{C}$ for $5 \mathrm{~min}$ and separated by SDS-PAGE before transfer of the semi-dry samples onto polyvinyl difluoride (PVDF) membranes. Western immunoblotting was carried out with anti-PDGFA (Bisso, bs-10073R, Beijing, China) primary antibodies at a 1:250 dilution, and samples were incubated for $2 \mathrm{~h}$ at $37^{\circ} \mathrm{C}$. After washing 3 times with Dulbecco's phosphate buffered saline (DPBS), the appropriate horseradish peroxidase-conjugated secondary antibody (Bisso, bs-0295D, Beijing, China) was incubated with the blot for $1 \mathrm{~h}$ at $37^{\circ} \mathrm{C}$, followed by washing 3 times in DPBS. The bands were visualised with a chemiluminescence detection kit (ECL, Rockford, IL, USA), and the result was analysed by ImageJ Software v. 1.38 (Windows version of NIH Image, http://rsb.info. nih.gov/nih-image/), Quantity One basic v. 4.6.3 (Bio-Rad Laboratories, Hercules, CA, USA), and MCID Analysis v. 7.0 (http://www.mcid.co.uk/software/mcid_analysis/ InterFocus Imaging, Linton, Cambridge, UK), according to protocols described by Gassmann [see 18]. The relative intensity of the target protein is proportional to the target band grey value/beta-actin band gradation value. Beta-actin was used as a loading control.

\section{Immunofluorescence analysis for PDGFA}

The skin brain tissue sections were incubated at room temperature in $3 \% \mathrm{H}_{2} \mathrm{O}_{2}$ for 10 min to block the action of any endogenous peroxidase. The immunofluorescence analysis for PDGFA was performed according to the manufacturer's instructions. Afterward, sections were incubated at $4^{\circ} \mathrm{C}$ overnight in the primary antibody solution with anti-anti-PDGFA (Bisso, bs-10073R, Beijing, China) (1:50). Following three 5 -min washes in $0.1 \mathrm{~mol} / \mathrm{L}$ phosphate buffered saline (PBS), sections were incubated with the secondary antibody, HRP-anti-rabbit IgG (Bisso, bs-0295D, Beijing, China, 1:100) for $30 \mathrm{~min}$ at $37^{\circ} \mathrm{C}$. After washing with $0.1 \mathrm{~mol} / \mathrm{L}$ PBS three additional times, $\mathrm{DAB}$ (3,3'-diaminobenzidine) staining was applied, using BSA instead of the primary antibody as a negative control in the reaction. Immunofluorescence was then observed under a fluorescence microscope at $400 x$ magnification (Olympus-71, Japan).

\section{Statistical analysis}

Data were expressed as the mean \pm standard error of the mean (SEM). Statistical analyses were performed using one-way ANOVA. All statistical computations were performed using SPSS software (version 13.0). The significance level was set at $p<0.05$. Protein abundance ( $n=3$ replicates) was determined by analysis of variance. Each experiment was repeated at least three times. 


\section{RESULTS}

\section{Characteristic of yak PDGFA gene}

The yak PDGFA cDNA sequence we obtained comprises 755 bp containing an open reading frame (ORF) of $636 \mathrm{bp}$, starting with an ATG start codon at position 2, and ending with a TAA stop codon at position 637, encoding 211 amino acid residues (Fig. 1). Our yak PDGFA cDNA sequence has been deposited in GenBank with accession no. KU851801.

Pairwise DNA sequence identities between yak and mouse, human, dog, pig, and sheep, PDGFA gene sequences are $83.8 \%, 86.2 \%, 86.8 \%, 91.3 \%$, and $93.8 \%$, respectively. Sequence identities between the yak and Asian buffalo, and against the cattle gene sequence are much higher, both $98.1 \%$ (Fig. 2). This highly conserved pattern of PDGFA evolution is displayed in Figure 3, by comparing the nucleotide and amino acid sequences of yak, cattle, and Asian buffalo. The multiple alignment of nucleotide and amino acid sequences shows 8 single nucleotide polymorphic (SNP) sites in the DNA (c. $270 \mathrm{~A}>\mathrm{G}, \mathrm{c} .290$ $\mathrm{T}>\mathrm{A}$, c. $451 \mathrm{~T}>\mathrm{A}$, c. $453 \mathrm{C}>\mathrm{G}$, c. $460 \mathrm{~T}>\mathrm{A}$, c. $462 \mathrm{~T}>\mathrm{G}$, and c. $561 \mathrm{G}>\mathrm{C}$ ) (Fig. 4A), which create four amino acid mutations (AA97 $M>K$, AA151 $Y>K, A A 154 Y>K$, and AA187 E > D) (Fig. 4B) in the ORF region of PDGFA gene between the three species.

\section{Relative expressions of yak PDGFA gene}

We measured the expression level of PDGFA mRNA in the cerebrum, cerebellum, hippocampus, and spinal cord by qRT-PCR (Fig. 5). The expression of PDGFA mRNA was the highest in the cerebrum and the lowest in the hippocampus; there was no significant difference between the hippocampus and spinal cord ( $p>0.05$ ) (Fig. 5). Relative PDGFA mRNA expression levels in the cerebrum and cerebellum were significantly higher than in hippocampus tissue ( $<0.05$ ) (Fig. 5).
GATGAGGACCTGGGCTTGTCTGCTGCTCCTCGGCTGCGGGTACCTC GCCAATGCCCTGGCCGAGGAAGCCGAGATCCCCCGCGAGGTCATT GAGAGGCTGGCGCACAGTCAGATCCACAGCATCCGGGACCTCCAG CGACTCCTGGAGATAGACTCCGTAGGAGCTGAGGAGCCTTTGGAA ACCAGTCTGAGAGCCCACGGGGGCCACGGCGCCAAGCATGCCCTG GAGAAGCGGCCAGTGCCCATCCGGAGGAAGAGGAGCATCGAGGA AGCCATCCCAGCGGTCTGCATGACCAGGACTGTCATTTACGAGATA CCTCGGAGCCAGGTGGACCCCACGTCCGCTAACTTCCTAATCTGGC CGCCGTGCGTGGAGGTGAAGCGCTGCACCGGCTGCTGCAACACAA GCAGCGTCAAGTGCCAGCCGTCGCGTGTCCACCACCGGAACGTCT ACGTGGCCTATGTGGAGTACTTCAGGAAGAAGGCGAAGTTAAAAG AGGTGCAGGTGCGGCTGGAGGAGCACCTGGAGTGCACGTGCACG AGCGCCAGCCCGAGCCCCGAGCACCGCGAGGAGGAGGCGGGAAG GCGTAGGGAGTCAGGTAAAAAACGGAAAAGAAAAAGGTTAAAAC CCACCTAACGTGGCCAACCAGATGTGAGGTGAAGATCAGCCAGCA GCCCTCTCCCGGGACACGGATGTACATGGCGTGTTACATTCCTGAA CCTACTATGTACGGTGCTTACTGCCAGCGTGGTCT

Figure 1. Yak platelet derived growth factor-alpha gene sequence. Nucleotide sequences in red font are the primers used for cDNA amplification. The ATG and TAA start and stop codons are also in red font, and indicated by yellow stripes.

\section{Yak PDGFA expression in protein level}

There was considerably more PDGFA protein in the cerebrum and cerebellum than in the hippocampus and spinal cord (Fig. 6A). The lowest level of PDGFA protein expression was in the hippocampus and spinal cord, with no significant difference between the two ( $p>0.05)$. PDGFA protein levels in the cerebrum and cerebellum tissues were significantly higher than in hippocampus tissue $(p<0.05)$ (Fig. 6B).

\section{Immunofluorescence staining of PDGFA}

PDGFA was primarily located in the nucleus of glial cells and pyramidal cells of yak cerebrum (Fig. 7A), in the cytoplasm of cerebellum Purkinje cells, and in glial and stromal cells of the cerebellum (Fig. 7B). A small amount was located in glial cells of the hippocampus (Fig. 7C). Negative results were obtained in yak spinal cord (Fig. 7D).

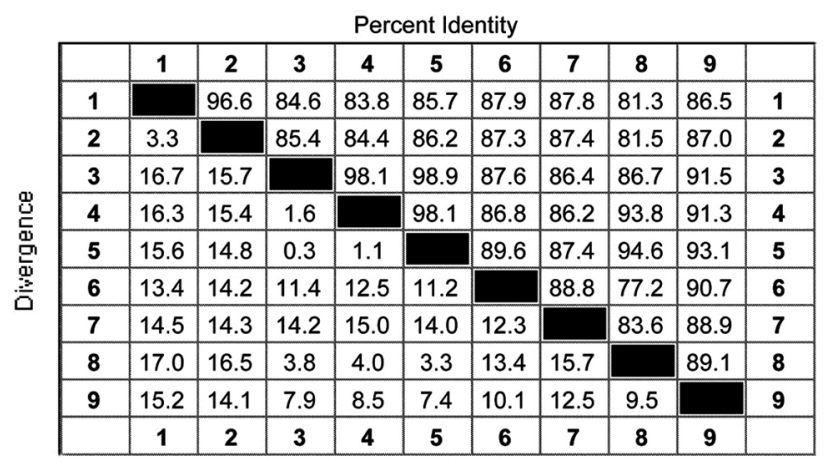

AY324648-Mus musculus BC061731-Rattus norvegicus JQ326282-Bubalus bubalis KU851801-Yak NM001075231-Bos taurus NM001190172-Canis lupus X03795-Human XM012132560-Ovis aries XM013987660-Sus scrofa

Figure 2. Similarity analysis of platelet derived growth factor-alpha gene sequence in yak and other species. 


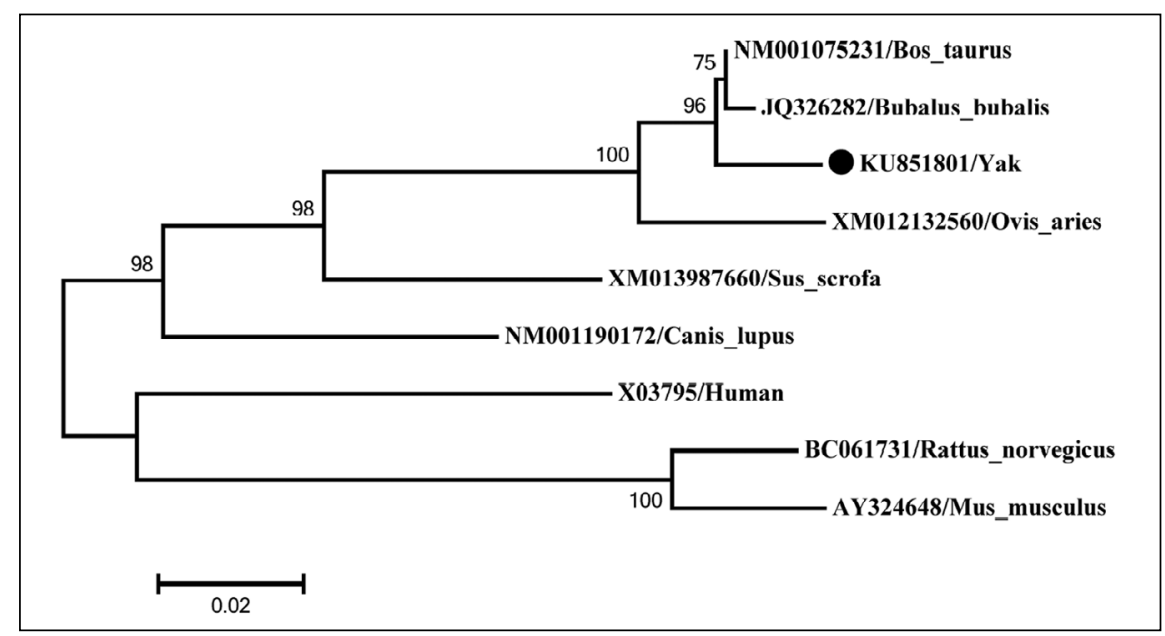

Figure 3. Phylogenetic analysis of platelet derived growth factor-alpha (PDGFA) gene sequence in yak and other species. Bootstrap values, expressed as percentages of 1,000 replicates, are given at the branch points. GenBank accession numbers and animal species are shown at each terminal node. The scale bar indicates nucleotide substitutions per site. The yak PDGFA gene sequence obtained in this study (GenBank: KU851801) is indicated by ' $\bullet$

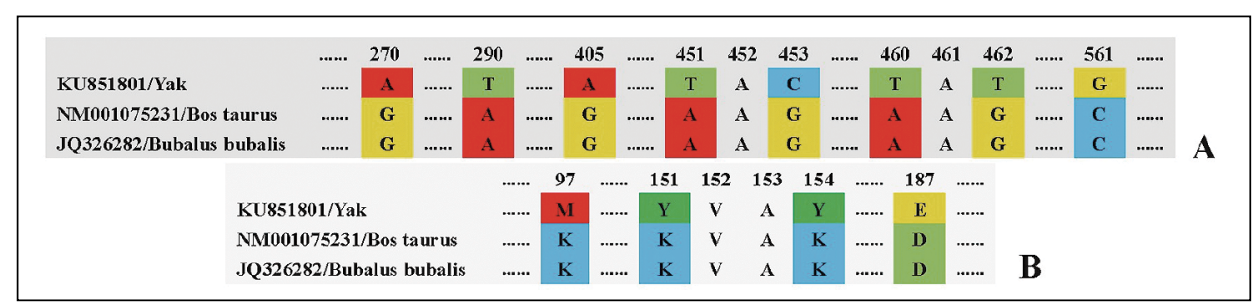

Figure 4. Multiple sequence alignment of yak nucleotide and amino acid sequence with Bubalus bubalus and Bos taurus; A. Multiple alignment of nucleotide sequences; $\mathbf{B}$. Multiple alignment of amino acid sequences.

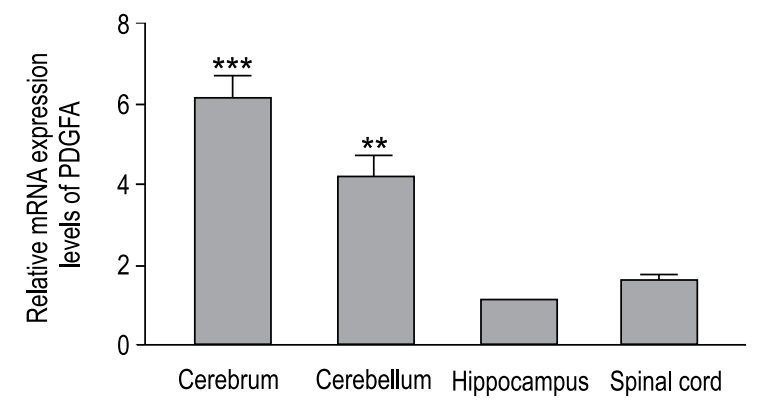

Figure 5. Platelet derived growth factor-alpha (PDGFA) mRNA quantitative real-time polymerase chain reaction results for different regions of yak brain tissue; ${ }^{* *} p<0.05,{ }^{* *} p<0.01$.

\section{DISCUSSION}

We successfully amplified a 755 bp yak PDGFA gene fragment that includes a $636 \mathrm{bp}$ ORF, encoding 211 amino acids (GenBank: KU851801). A multiple

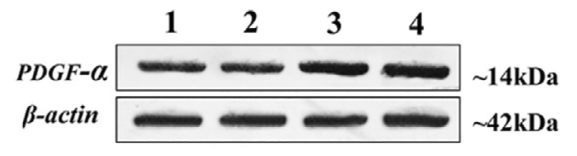

A

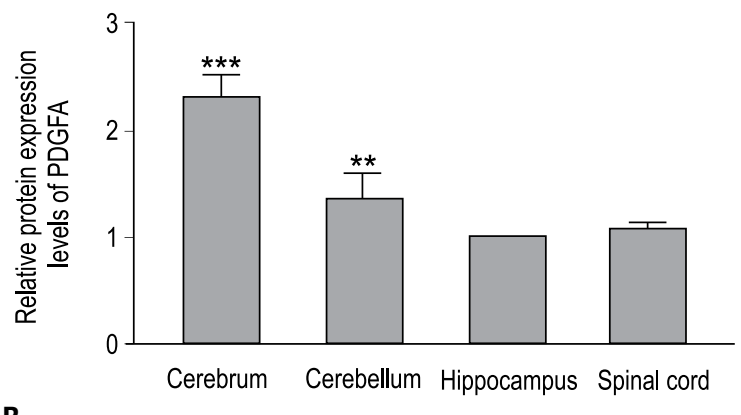

Figure 6. Detection of platelet derived growth factor-alpha (PDGFA) protein expression in yak brain tissue; $\mathbf{A}$. Western blot results for PDGFA and beta-actin protein in yak brain tissue; $1-4-$ samples from cerebrum, cerebellum, hippocampus, and spinal cord; B. Relative expression levels of PDGFA protein in different regions of yak brain tissue; ${ }^{* *} \mathrm{p}<0.05,{ }^{* * *} \mathrm{p}<0.01$. 

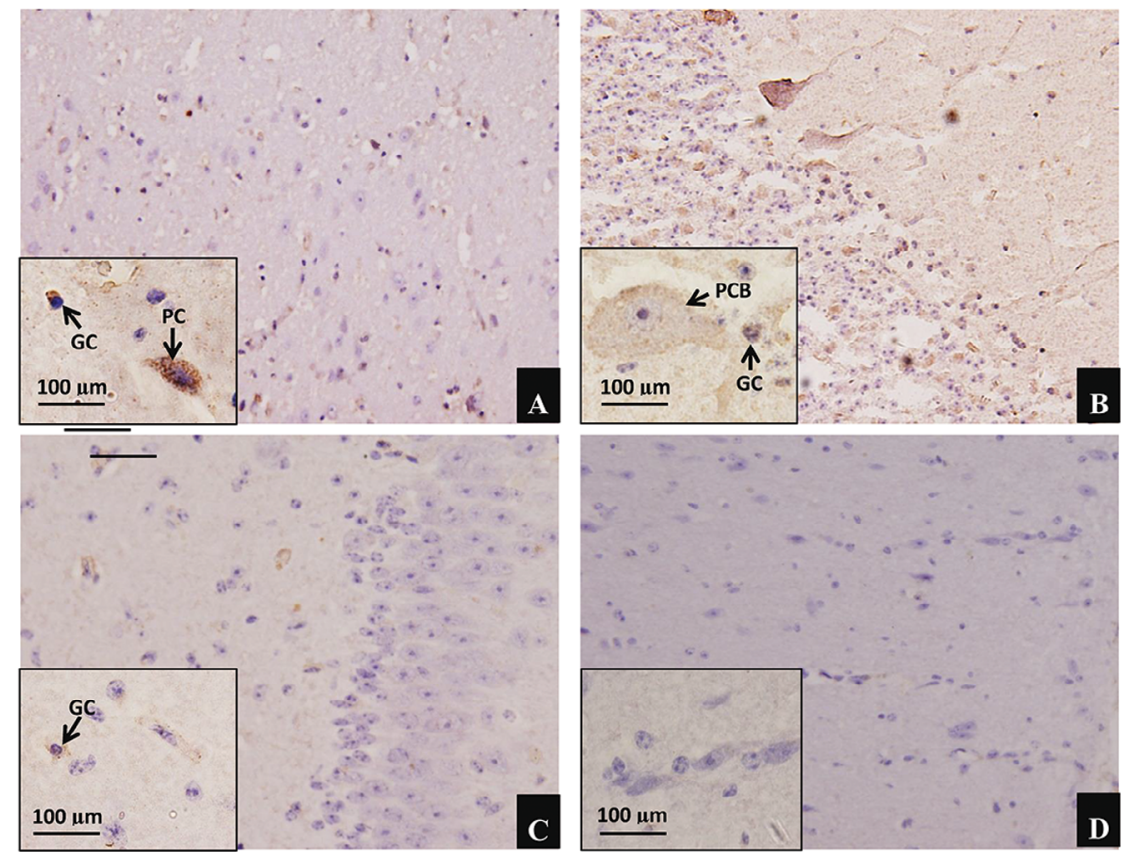

Figure 7. Immunohistochemistry of platelet derived growth factor-alpha (PDGFA) in yak brain tissue $(200 \times)$; A. Expression of PDGFA in yak cerebrum; B. Expression of PDGFA in yak cerebellum; C. Expression of PDGFA in yak hippocampus; D. Expression of PDGFA in yak spinal cord; GC — glial cell; PC — pyramidal cell; PCB — Purkinje cell body.

sequence alignment between the yak PDGFA nucleotide and amino acid sequence with the cattle and Asian buffalo sequences shows 8 SNP sites (Fig. 4A), resulting in 4 amino acid substitutions (Fig. 4B). These variations may have important biological significance, perhaps conferring PDGFA functional physiological advantages under hypoxic conditions. The expression of PDGFA in other animals has been shown to relate to environmental stress, including hypoxia [23]; therefore, the unique characteristics of yak PDGFA could be a crucial factor in high altitude hypoxia adaption.

PDGFs, as key autocrine and paracrine growth factors, play important roles in embryonic development, particularly those that promote the development of the CNS and related organs [9]. PDGFA contributes to CNS oligodendrocyte growth and nuclear proliferation under standard physiological conditions $[1,26]$. Our results show that yak brain cerebrum, cerebellum, hippocampus, and spinal cord tissues all express the PDGFA gene and its protein at varying levels, and are widely expressed throughout various parts of the adult yak CNS under normal physiological conditions. The roles of PDGFs in adult animals' nervous systems should not be ignored. Whether the differences in PDGFA gene and protein expression levels between different tissues of the brain relate to yak's long-term physiological adaptabilities in hypoxic environments remains a question. Further study requires a more detailed examination of yak PDGFA gene specificity.

Our immunohistochemistry results show the PDGFA protein to be highly expressed in yak cerebrum tissue glial cells and pyramidal cells, and especially so in Purkinje cells, as well as in cerebellum and hippocampus tissues, but considerably less in spinal cord tissues, under normal physiological conditions. This is consistent with previous studies $[22,25]$. The PDGFA protein mainly occurs in CNS glial cells and neurons. We confirmed the distribution of PDGFA in the cerebrum tissue of yak pyramidal cells and Purkinje cells. This particular expression pattern has not been reported in other species. PDGFA protein in the spinal cord could be detected by western blot, but not by immunohistochemistry, which may be due to its similarity to VEGF proteins $[8,24]$. Our present study only focused on nerve cells, and PDGFA may also be expressed in vascular endothelial cells. Our team is currently working on this subsequent analysis in yak.

Other members of the PDGFs family exist in addition to PDGFA, including PDGFB $[8,14]$. These growth factors and the associated receptors, not only play important biological roles in embryonic development and organ 
formation [13], but also affect the physiology of adult animals, including neuroprotective and anti-tumour effects $[1,20]$. However, the molecular mechanism and transcriptional regulation of various PDGF family members vary $[6,14]$. Therefore, whether other PDGF family members participate in the regulation of yak physiological function should be further explored.

\section{CONCLUSIONS}

Our study shows the PDGFA gene to be highly evolutionarily conserved, with high sequence identities within mammals. Nonetheless, the yak PDGFA gene and its protein have several species-specific characteristics. The yak PDGFA gene and its protein are widely expressed in the yak brain. However, differences in yak PDGFA expression levels in the cerebrum, cerebellum, hippocampus, and spinal cord suggest that PDGFA may affect adaptive physiological functions in the yak, and require further study.

\section{Acknowledgements}

The present study was supported by the National Natural Science Foundation of China (Grant No. 31572478).

\section{REFERENCES}

1. Andrae J, Gallini R, Betsholtz C. Role of platelet-derived growth factors in physiology and medicine. Genes Dev. 2008; 22(10): 1276-1312, doi: 10.1101/gad.1653708, indexed in Pubmed: 18483217.

2. Ball SG, Shuttleworth CA, Kielty CM. Vascular endothelial growth factor can signal through platelet-derived growth factor receptors. J Cell Biol. 2007; 177(3): 489-500, doi: 10.1083/jcb.200608093, indexed in Pubmed: 17470632.

3. Basciani S, Mariani S, Arizzi M, et al. Expression of platelet-derived growth factor-A (PDGF-A), PDGF-B, and PDGF receptor-alpha and -beta during human testicular development and disease. J Clin Endocrinol Metab. 2002; 87(5): 2310-2319, doi: 10.1210/ /jcem.87.5.8476, indexed in Pubmed: 11994382.

4. Basciani S, Mariani S, Spera G, et al. Role of platelet-derived growth factors in the testis. Endocrine Rev. 2010; 31(6): 916-939, doi: 10.1210/er.2010-0004.

5. Betsholtz C, Karlsson L, Lindahl P. Developmental roles of platelet-derived growth factors. Bioessays. 2001; 23(6): 494-507, doi: 10.1002/bies.1069, indexed in Pubmed: 11385629.

6. Bhandari B, Grandaliano G, Abboud HE. Platelet-derived growth factor (PDGF) BB homodimer regulates PDGF A- and PDGF B-chain gene transcription in human mesangial cells. Biochem J. 1994; 297 (Pt 2): 385-388, indexed in Pubmed: 8297346.

7. Demaria M, Ohtani N, Youssef SA, et al. An essential role for senescent cells in optimal wound healing through secretion of PDGF-AA. Dev Cell. 2014; 31(6): 722-733, doi: 10.1016/j.devcel.2014.11.012, indexed in Pubmed: 25499914.

8. Fredriksson L, Li H, Eriksson U. The PDGF family: four gene products form five dimeric isoforms. Cytokine Growth Factor Rev. 2004; 15(4): 197-204, doi: 10.1016/j.cytogfr.2004.03.007, indexed in Pubmed: 15207811.

9. Fruttiger M, Karlsson L, Hall AC, et al. Defective oligodendrocyte development and severe hypomyelination in PDGF-A knockout mice. Development. 1999; 126(3): 457-467, indexed in Pubmed: 9876175.

10. Gnessi L, Basciani S, Mariani S, et al. Leydig cell loss and spermatogenic arrest in platelet-derived growth factor (PDGF)-Adeficient mice. J Cell Biol. 2000; 149(5): 1019-1026, indexed in Pubmed: 10831606.

11. He Y, Yu S, Hu J, et al. Changes in the Anatomic and Microscopic Structure and the Expression of HIF-1alpha and VEGF of the Yak Heart with Aging and Hypoxia. PLoS One. 2016; 11(2): e0149947, doi: 10.1371/journal.pone.0149947, indexed in Pubmed: 26914488.

12. Heldin $\mathrm{CH}$, Westermark B. Mechanism of action and in vivo role of platelet-derived growth factor. Physiol Rev. 1999; 79(4): 1283-1316, indexed in Pubmed: 10508235.

13. Hoch RV, Soriano P. Roles of PDGF in animal development. Development. 2003; 130(20): 4769-4784, doi: 10.1242/dev.00721, indexed in Pubmed: 12952899.

14. Johnsson A, Heldin $\mathrm{CH}$, Westermark $\mathrm{B}$, et al. Platelet-derived growth factor: identification of constituent polypeptide chains. Biochem Biophys Res Commun. 1982; 104(1): 66-74, indexed in Pubmed: 7073684.

15. Kaetzel D. Transcription of the platelet-derived growth factor A-chain gene. Cytokine Growth Factor Rev. 2003; 14(5): 427-446, doi: 10.1016/s1359-6101(03)00051-0.

16. Klinghoffer RA, Hamilton TG, Hoch $R$, et al. An allelic series at the PDGFalphaR locus indicates unequal contributions of distinct signaling pathways during development. Dev Cell. 2002; 2(1): 103-113, indexed in Pubmed: 11782318.

17. Kohler N, Lipton A. Platelets as a source of fibroblast growthpromoting activity. Exp Cell Res. 1974; 87(2): 297-301, indexed in Pubmed: 4370268.

18. Max G, Beat G, Bianca R, et al. Quantifying Western blots: pitfalls of densitometry. Electrophoresis. 2009; 30(11): 1845-1855, doi: 10.1002/elps.200800720, indexed in Pubmed: 19517440.

19. McDonald NQ, Hendrickson WA. A structural superfamily of growth factors containing a cystine knot motif. Cell. 1993; 73(3): 421-424, indexed in Pubmed: 8490958.

20. Narita M, Usui A, Narita M, et al. Protease-activated receptor-1 and platelet-derived growth factor in spinal cord neurons are implicated in neuropathic pain after nerve injury. J Neurosci. 2005; 25(43): 10000-10009, doi: 10.1523/JNEUROSCI.2507-05.2005, indexed in Pubmed: 16251448.

21. Pfaffl MW, Horgan GW, Dempfle L. Relative expression software tool (REST) for group-wise comparison and statistical analysis of relative expression results in real-time PCR. Nucleic Acids Res. 2002; 30(9): e36, indexed in Pubmed: 11972351.

22. Pringle NP, Richardson WD. A singularity of PDGF alpha-receptor expression in the dorsoventral axis of the neural tube may define the origin of the oligodendrocyte lineage. Development. 1993; 117(2): 525-533, indexed in Pubmed: 8330523.

23. Ross R, Glomset J, Kariya B, et al. A platelet-dependent serum factor that stimulates the proliferation of arterial smooth muscle cells in vitro. Proc Natl Acad Sci U S A. 1974; 71(4): 1207-1210, indexed in Pubmed: 4208546.

24. Tarsitano M, De Falco S, Colonna V, et al. The C. elegans pvf-1 gene encodes a PDGF/VEGF-like factor able to bind mammalian VEGF receptors and to induce angiogenesis. FASEB J. 2006; 20(2): 227-233, doi: 10.1096/fj.05-4147com, indexed in Pubmed: 16449794.

25. Westermark $B$, Wasteson $A$. A platelet factor stimulating human normal glial cells. Exp Cell Res. 1976; 98(1): 170-174, indexed in Pubmed: 1253836.

26. Woodruff RH, Fruttiger M, Richardson WD, et al. Platelet-derived growth factor regulates oligodendrocyte progenitor numbers in adult CNS and their response following CNS demyelination. Mol Cell Neurosci. 2004; 25(2): 252-262, doi: 10.1016/j. mcn.2003.10.014, indexed in Pubmed: 15019942.

27. Yang X, Ren X, Yu S, et al. Morphological study of the hoof in yak. Folia Morphol. 2016; 75(3): 400-408, doi: 10.5603/ FM.a2015.0132, indexed in Pubmed: 26711656. 